\title{
HISTOPATHOLOGICAL STUDY OF NEOPLASTIC AND NON-NEOPLASTIC THYROID LESIONS: AN INSTITUTIONAL EXPERIENCE OF 2 YEARS
}

\author{
Monalisa Dash', K. P. A. Chandrashekhar², K. Raghu³, Saadvi Kethireddy 4 \\ ${ }_{1}^{1}$ Postgraduate, Department of Pathology, SVS Medical College, Mahabubnagar, Telangana State. \\ 2 Professor, Department of Pathology, SVS Medical College, Mahabubnagar, Telangana State. \\ 3 Professor, Department of Pathology, SVS Medical College, Mahabubnagar, Telangana State. \\ ${ }^{4}$ Postgraduate, Department of Pathology, SVS Medical College, Mahabubnagar, Telangana State.
}

\begin{abstract}
BACKGROUND

Thyroid is the first endocrine gland to develop in the foetal life; superficial in location, amenable to direct physical examination and biopsy. Disorders of thyroid include a vast array of genetic, inflammatory, developmental, immunologic \& neoplastic disorders. The non-neoplastic lesions of thyroid constitute a major part of the cases of thyroid in hospitals.
\end{abstract}

\section{AIMS AND OBJECTIVES}

To study the prevalence of both neoplastic and non-neoplastic lesions of thyroid and the prevalence and histological changes according to age, gender and severity.

\section{MATERIALS AND METHODS}

The duration of the study is 2 years [June 2013 to May 2015]. A review of histopathological reports of all thyroid specimens received in the Department of Pathology, SVS Medical College, Mahabubnagar for a period of 2 years were done. The specimens included were of lobectomy, hemithyroidectomy, and also subtotal and near-total thyroidectomy. A total of 150 thyroid specimens were analysed. Thyroid specimen was immediately fixed into $10 \%$ formalin and processed by paraffin embedding. Sections were stained by Haematoxylin and eosin stain and microscopic examination was done.

\section{RESULTS}

One hundred and fifty thyroidectomy specimens were reviewed. Multinodular goitre was the commonest non-neoplastic thyroid lesion constituting 52 cases. Follicular adenoma was the most common benign neoplasm (33 cases out of 40 neoplastic lesions). Papillary carcinoma was the most common malignant neoplasm. Peak incidence of thyroid lesions was seen in the $3^{\text {rd }}$ to $4^{\text {th }}$ decade and more common in females.

\section{CONCLUSION}

Thyroid nodules which occur in 4 to $7 \%$ of adults are a source of concern for patients and a diagnostic dilemma for the clinicians. The final answer is histopathological examination or fine needle aspiration cytology. Our study which was carried out in a te aching hospital showed goitre as the commonest lesion.

\section{KEYWORDS}

Follicular Adenoma, Multinodular Goitre, Papillary Carcinoma Thyroid.

HOW TO CITE THIS ARTICLE: Dash M, Chandrasekhar KPA, Raghu K, et al. Histopathological study of neoplastic and nonneoplastic thyroid lesions: An institutional experience of 2 years. J. Evolution Med. Dent. Sci. 2016;5(73):5348-5351, DOI: $10.14260 /$ jemds/2016/1213

\section{INTRODUCTION}

Thyroid diseases are quite common worldwide and are one of the common problems encountered in clinical practice with majority of them benign in nature. The enlargement of thyroid gland is a major health concern in many parts of the world. Despite its structural simplicity, the thyroid gland can give rise to a large variety of pathological lesions, both neoplastic and non-neoplastic which usually present clinically as diffuse swelling or as a discrete palpable solitary nodules.

Financial or Other, Competing Interest: None.

Submission 06-08-2016, Peer Review 30-08-2016,

Acceptance 06-09-2016, Published 10-09-2016.

Corresponding Author:

Dr. Monalisa Dash,

C/o. Dr. Jugajyoti Pathi

Administrative Officer,

Kalinga Institute of Dental Sciences,

Bhubaneswar.

E-mail: monalisha89@gmail.com

DOI: $10.14260 /$ jemds $/ 2016 / 1213$
Today, thyroidectomy is a routine procedure because of the introduction of safe anaesthesia, antiseptic, fine surgical instruments and development of new techniques offering the chances to cure many patients 1,2

Thyroid gland is the largest of all endocrine glands and because of its superficial location it is amenable to direct physical examination and biopsy as well as fine needle aspiration cytology (FNAC). Thyroid lesions may be developmental, inflammatory, hyperplastic or neoplastic. Thyroid disease can lead to systemic diseases like Graves' disease or a local abnormality like multinodular goitre or benign follicular adenoma.

Thyroid carcinoma mostly resembles its benign counterpart in physical characteristics, physiological parameters such as T3 and T4 levels. Therefore, the surgical excision of the nodule and its histological examination is the only way to differentiate between the more frequent benign lesion and much less malignant nodules. A solitary thyroid nodule is defined as palpable single clinically detected nodule in the thyroid. It causes more concern because of high 
probability of malignancy. Thyroid cancers are second most common cancers in female patients after breast cancers. ${ }^{3}$

Diffuse thyroid lesions are those that are associated with conditions affecting entire glands such as hyperplasia and thyroiditis. The most common non-neoplastic thyroid lesion is goitre. It may be colloid or nodular goitre or simple goitre. It could be due to hyperthyroidism or hypothyroidism. The common cause of hyperthyroidism is Graves' disease. Hashimoto's thyroiditis is the predominant inflammatory lesion while subacute lymphocytic thyroiditis, subacute granulomatous thyroiditis, post-partum thyroiditis comprise a minor proportion. Goitre is an enlargement of the thyroid gland that often produces a significant swelling in front of the neck. This enlargement can be caused by iodine deficiency, inability of the body to use iodine correctly or a variety of thyroid disorder including infections, tumours and autoimmune diseases. Some environmental pollutants, heavy metals, metal poisoning and certain drugs can also contribute to goitre formation.

Iodine deficiency goitre can be common in regions where the soil and foods have insufficient iodine. Preschool children, adolescent girls, pregnant women and the elderly are more vulnerable to goitre and other iodine deficiency disorders. Areas where iodine supplies are inadequate show high rates not only of goitre but also of birth defects and mental and physical retardation. This study was conducted to see the frequency of different thyroid lesions on clinicopathological ground.

Nodular lesion comprises those disorders that produce a clinical nodule and consists of non-neoplastic hyperplasia as well as benign and malignant tumours. Thyroid nodules which occur spontaneously in 4 to $7 \%$ of adult population are source of concern for patients and a diagnostic dilemma for the clinicians.

Neoplasms of thyroid are fairy uncommon. They constitute only $0.7 \%$ of all cancers in female and $0.2 \%$ in males. There are different diagnostic modalities used to evaluate and diagnose thyroid nodules efficiently. These include clinical examination, thyroid function test, and ultrasonography, scintiscan, fine needle aspiration cytology and histopathological examination.

However, final diagnosis requires morphological examination. Fine needle aspiration cytology is time saving and cost effective, but it has its own limitations and disadvantages like morphology of some tumours which is not clear. Thus, a specific diagnosis can only be arrived at after a histological examination.

\section{MATERIALS AND METHODS}

A total of one hundred and fifty surgical specimens of patients were sent to the department of pathology for histopathological examination in last 2 years' span. The procedures undertaken to obtain these specimens were lobectomy, hemithyroidectomy, subtotal and near-total thyroidectomy. All the specimens were fixed in 10\% Formalin and were processed to make paraffin embedded tissue blocks and then sectioned. All specimen sections were stained with haematoxylin and eosin and where ever necessary stained with special stains like Congo red as in medullary carcinoma of thyroid to show the presence of Amyloid. Strict morphologic diagnostic criteria were used for the diagnosis of all the pathologies. Data were obtained and subjected to statistical analysis.

\section{RESULTS}

One hundred and fifty thyroidectomy specimens were reviewed. The age and sex distribution of patients is shown in tables 1 and $2 \& 3$. One hundred and thirty eight cases were females and twelve cases were males. Table 4 shows the different thyroid diseases. Multinodular goitre is the commonest in non-neoplastic lesions of thyroid, 52 cases (47.3\%). Papillary carcinoma was the commonest malignant tumour in this study ( 4 cases). In malignant neoplastic of thyroid lesion category only two cases reported as follicular variant of papillary carcinoma of thyroid gland as well as only one case reported as medullary carcinoma of thyroid gland. 14 cases of Hashimoto's thyroiditis were found in patients studied and all were females (100\%), the age ranged between 21-40 years. Rest of 33 cases were benign follicular adenomas and were showing female predominance in this study. Table 2 shows the age range and gender distribution of different types of thyroid diseases diagnosed during this study. The age ranged between 00-79 years in both males and females. The most common age ranged between 31-40 years in both males and females in non-neoplastic thyroid lesions. Only one case $(0.9 \%)$ of lymphocytic thyroiditis was reported.

Incidence of non-neoplastic thyroid lesions was 73.3\% while that of neoplastic lesions was $26.3 \%$. Middle aged patients $31-40$ years (36.3\%) and females (90.9\%) were more commonly affected in benign lesions. Amongst nonneoplastic lesions, multinodular goitre (47.3\%) was commonest while papillary carcinomas of thyroid (57.1\%) were common malignant lesions.

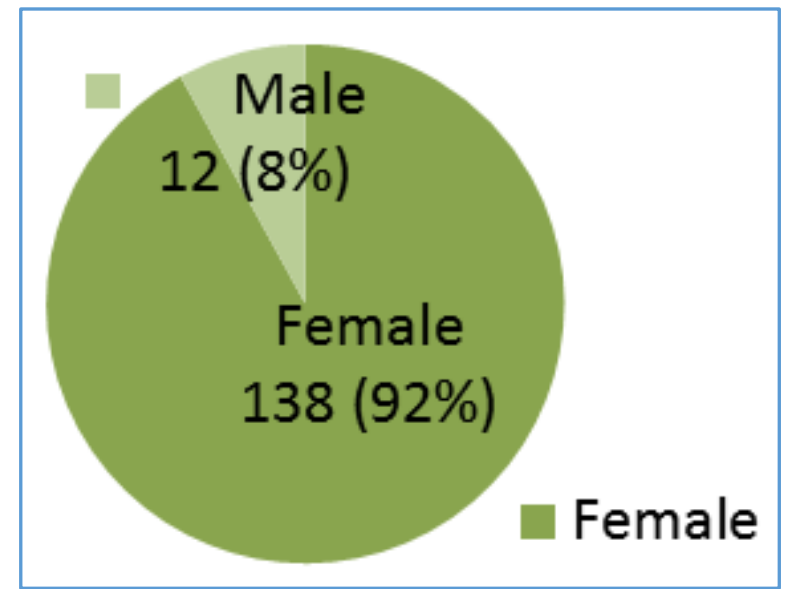

Fig. 1: Showing Gender Distribution of Thyroid Lesions

\begin{tabular}{|c|c|c|}
\hline Age & No. of Cases & (\%) \\
\hline $0-10$ & 00 & 00 \\
\hline $11-20$ & 4 & 3.6 \\
\hline $21-30$ & 32 & 29.1 \\
\hline $31-40$ & 33 & 30 \\
\hline $41-50$ & 22 & 20 \\
\hline $51-60$ & 10 & 9.1 \\
\hline $61-70$ & 9 & 8.2 \\
\hline $71-79$ & 00 & 00 \\
\hline \multicolumn{2}{|c|}{ Fig. 2: Showing Age Distribution in Non-Neoplastic } \\
Thyroid Lesions \\
\hline
\end{tabular}




\begin{tabular}{|c|c|c|c|c|}
\hline Age & Malignant & $\mathbf{( \% )}$ & Benign & $\mathbf{( \% )}$ \\
\hline $0-10$ & 00 & 00 & 00 & 00 \\
\hline $11-20$ & 00 & 00 & 03 & 9.1 \\
\hline $21-30$ & 00 & 00 & 08 & 24.2 \\
\hline $31-40$ & 00 & 00 & 12 & 36.3 \\
\hline $41-50$ & 04 & 57.1 & 04 & 12.1 \\
\hline $51-60$ & 02 & 28.6 & 03 & 9.1 \\
\hline $61-70$ & 01 & 14.3 & 02 & 6.1 \\
\hline $71-79$ & 00 & 00 & 01 & 3.1 \\
\hline TOTAL & 07 & 100 & 33 & 100 \\
\hline \multicolumn{5}{|c|}{ Fig. 3: Showing Age Distribution of Both Benign } \\
and Malignant Thyroid Lesions \\
\hline
\end{tabular}

\begin{tabular}{|c|c|c|}
\hline Non Neoplastic & No. of Cases & $\mathbf{( \% )}$ \\
\hline Simple Goitre & 43 & 39.1 \\
\hline MNG & 52 & 47.3 \\
\hline Hashimoto Thyroiditis & 14 & 12.7 \\
\hline Lymphocytic Thyroiditis & 01 & 0.9 \\
\hline Total & $\mathbf{1 1 0}$ & $\mathbf{1 0 0}$ \\
\hline LESION & No. of Cases & $\mathbf{( \% )}$ \\
\hline Papillary Carcinoma Thyroid & 4 & 57.1 \\
\hline $\begin{array}{c}\text { Follicular Variant of Papillary } \\
\text { Carcinoma Thyroid }\end{array}$ & 2 & 28.6 \\
\hline Medullary Carcinoma Thyroid & 1 & 14.3 \\
\hline \multicolumn{2}{|c|}{ Total } & $\mathbf{7}$ \\
\hline $\begin{array}{c}\text { Fig. 4: Showing Distribution of Both Non-Neoplastic } \\
\text { and Neoplastic Thyroid Lesions }\end{array}$ \\
\hline \multicolumn{2}{|c}{} \\
\hline
\end{tabular}

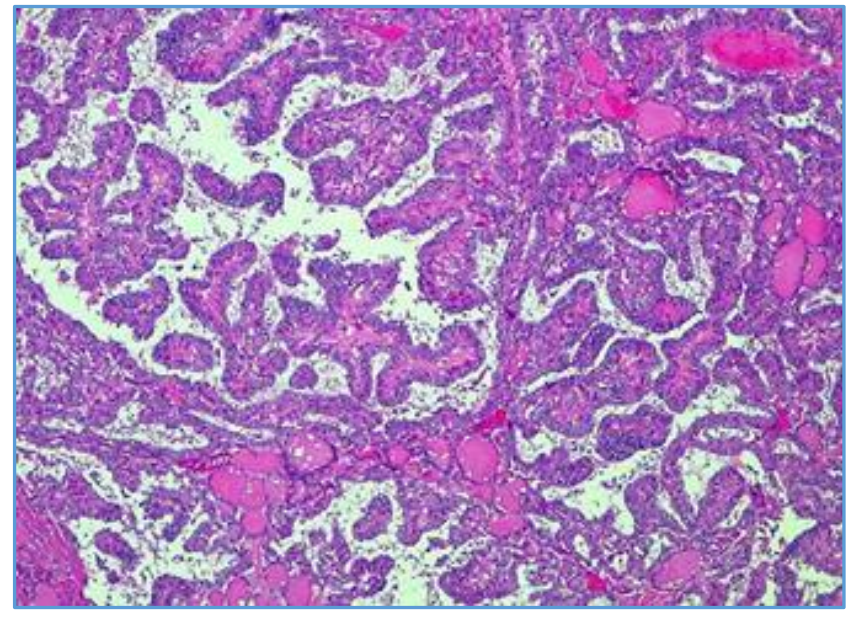

Papillary Carcinoma of Thyroid (10x)

\section{DISCUSSION}

Diseases of the thyroid are of great importance because most are amenable to medical or surgical management. It is known that approximately $1-10 \%$ of adults in the USA are reported to have solitary nodules. ${ }^{4}$ This incidence is even significantly higher in endemic goitrous regions. Single nodules are about four times more common in women than in men and this incidence increases throughout life. In fact, benign neoplasms outnumber thyroid carcinomas by a ratio of nearly $10: 12^{2,4}$ Overall, the incidence of thyroid malignancy is low, forming $0.5-1.0 \%$ of all cancers and $3.3-17 \%$ of all thyroid diseases. 4 Carcinomas of the thyroid are thus uncommon, accounting for less than $1 \%$ of solitary thyroid nodules and representing about 15,000 new cancer cases in North America each year. 4,5 Moreover, most thyroid cancers are indolent, permitting $90 \%$ survival at 20 years. Thyroidectomy specimens in this study formed approximately $2 \%$ of all surgical pathology load received at the department of pathology during the study period. Therefore, it is not uncommon and much concordant with most pathology practices in North America and Europe ${ }^{6-}$ 7. In this study, multinodular goitre accounted for $47.3 \%$ (Fifty two cases) forming the most common pathologic presentation with a peak in $2^{\text {nd }}$ to $4^{\text {th }}$ decades and female preponderance. Clinically, 42 cases presented with nodular enlargement and 10 cases presented with diffuse enlargement of the gland and 12 cases presented with Hashimoto's thyroiditis. All the cases were females and age distribution was in between 20-30 years of age. All cases presented with diffuse swelling of thyroid glands.

Thyroid malignancy is represented exclusively in this study by papillary carcinoma only. It ranks the most common presentation as a solitary nodule forming $57.1 \%$ (4 cases). Females were affected more compared to males. Other types of thyroid cancer diagnosed during this study were follicular variant of papillary thyroid carcinoma followed by medullary carcinoma of thyroid. The alarming fact is the higher frequency of papillary carcinoma that needs further investigation. Dietary factors may play a role; however, exposure to low doses of diagnostic irradiation or following exposure to radioactive substances still needs to be ruled out. As in most reported cases of papillary carcinoma, this study has shown females constituted more. Age is one of the most significant prognostic factors in papillary carcinoma of thyroid. Clinically, patients presented with nodular swelling of the thyroid gland. Hormonal studies were within normal 
limits. Lymph node involvement was seen in 2 cases. Occasionally, a patient may present with obvious metastatic disease with an undetectable primary that is called occult or hidden malignancy of thyroid gland and mostly seen in papillary carcinoma of thyroid. Mortality is very rare in patients under the age of forty years. The fact that most of the papillary carcinomas occur in patients less than forty years of age, as seen in the present study may explain the better prognosis. No case of follicular carcinoma was seen in this study. The incidence of follicular carcinoma is increased in areas of dietary iodine deficiency, suggesting that in some cases, multinodular goitre may predispose to the development of the neoplasm. Mostly follicular carcinoma shows haematogenous spread. Benign adenomas (Using strict diagnostic criteria) form $82.5 \%$ (33 cases).

Follicular adenomas may appear in any age group, but most common in middle age, they are several times more common in females than in males as it has been observed in the present study and also by Evans $\mathrm{HL}^{8}$ and Ljungberg $0 .^{9}$

\section{CONCLUSION}

Most of the patients presented with symptoms of neck swelling. In this study, a high female predilection was noticed. Peak age of incidence of thyroid lesions was found out to be 20-40 years. Most common non-neoplastic lesion encountered is multinodular goitre and most common malignant neoplastic lesion encountered is papillary carcinoma thyroid whereas most common benign neoplastic lesion is adenoma thyroid. Microscopic examination remained the gold standard for the ultimate and accurate diagnosis. The present study was undertaken to review the recent literature in recognising the histomorphologic criteria for the thyroid lesion with age and sex of patient in and around Mahabubnagar. The major drawback of this study was that the present data being hospital generated cannot be regarded as a representation of the incidence of thyroid lesions in general population. Similar studies will definitely be eye openers in planning and provisioning in health sector.

\section{REFERENCES}

1. Stewart BW, Kleihues P. World Cancer Report. Lyon: IARC Press 2003.

2. Burguera B, Gharib $H$. Thyroid incidentalomas. Prevalence, diagnosis, significance and management. Endocrinol Metab Clin North Am 2000;29(1):187-203.

3. Hussain N, Anwar M, Nadia N, et al. Pattern of surgically treated thyroid disease in Karachi. Biomedica 2005; 21:18-20.

4. Mackenzie EJ, Mortimer RH. Thyroid nodules and thyroid cancer. Med J Aust 2004;180(5):242-7.

5. Gharib H, Papini E, Vaicavi R, et al. AACE/AME medical guidelines for clinical practice for the diagnosis and management of thyroid nodules. Endocr Pract 2006; 12(1):63-102.

6. Welker MJ, Orlov D. Thyroid nodules. Am Fam Physician 2003;67(3):559-66.

7. Elhamel A, Sherif IH, Wassef SA. The pattern of thyroid disease in a closed community of $1-1 / 2$ million people. Saudi Med J 1988;9(5):481-4.

8. Evans HL. Follicular neoplasms of the thyroid-a study of 44 cases followed for a minimum of 10 years, with emphasis on differential diagnosis. Cancer 1984; 54(3):535-40.

9. Ljungberg 0. Biopsy pathology of the thyroid and parathyroid. London: Chapman \& Hall Medical publishing 1992. 